\title{
MiR-873-5p targets THUMPD1 to inhibit gastric cancer cell behavior and chemoresistance
}

\author{
Qinggui Chen ${ }^{1 \#}$, Li Lin ${ }^{2 \#}$, Boliang Xiong ${ }^{3 \#}$, Wensheng Yang ${ }^{4}$, Junli Huang ${ }^{5}$, Huibo Shi ${ }^{6,7}$, Zhenfa Wang ${ }^{2}$ \\ ${ }^{1}$ Department of General Surgery, Zhongshan Hospital, Xiamen University, Xiamen, China; ${ }^{2}$ Department of Gastrointestinal Surgery, Zhongshan \\ Hospital, Xiamen University, Xiamen, China; ${ }^{3}$ Department of Radiotherapy, Zhongshan Hospital, Xiamen University, Xiamen, China; ${ }^{4}$ Department \\ of Pathology, Chenggong Hospital, Xiamen University, Xiamen, China; ${ }^{5}$ Department of General Surgery, Chenggong Hospital, Xiamen University,

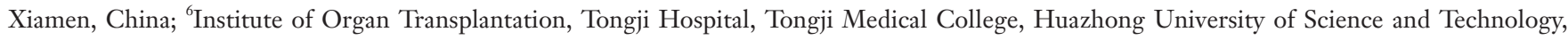 \\ Wuhan, China; ${ }^{7}$ Key Laboratory of Organ Transplantation, Ministry of Education NHC Key Laboratory of Organ Transplantation, Wuhan, China \\ Contributions: (I) Conception and design: None; (II) Administrative support: None; (III) Provision of study materials or patients: None; (IV) \\ Collection and assembly of data: Q Chen, L Lin, B Xiong; (V) Data analysis and interpretation: Q Chen, L Lin, B Xiong; (VI) Manuscript writing: \\ All authors; (VII) Final approval of manuscript: All authors. \\ "These authors contributed equally to this work and should be regarded as co-first authors. \\ Correspondence to: Zhenfa Wang. 201-209 Hubin South Road, Siming District, Xiamen 361004, China. Email: 13806062519@139.com; Huibo Shi. \\ Tongji Hospital, Tongji Medical College, Huazhong University of Science and Technology, Wuhan, 430030, China. Email: jimshihuihuibo@163.com.
}

Background: Gastric cancer is one of the most common gastrointestinal tumors. Evidence has pointed to the fact that miRNAs play critical roles in the occurrence, development, and metastasis of gastric cancer by regulating cell proliferation, differentiation, apoptosis, and invasion.

Methods: In this study, first the relationship of miR-873-5p level and tissues types/LN(+/-)/metastasis(+/-)/ tumor size was analysis, respectively. Second, the CCK8 and Transwell assay was used to determine the proliferation, invasion and migration of GC cells transfected with overexpression-/low expressionmiR-873-5p. Third, the cell viability were analysis in the GC cells transfected with overexpression-/low expression-miR-873-5p treatment with different chemotherapy drugs. Fourth, the target gene of miR-873$5 p$ was predicted using bioinformation methods. Fifth, the relationship of miR-873-5p with target geneTHUMPD1 were explored by using $\mathrm{Wb}$ and luciferase activity assay, et al.

Results: We confirmed that miR-873-5p was negatively correlated with GC including tumor size, LN metastasis, distant metastasis. The miR-873-5p enhanced the sensitivity of Doxorubicin/Fluorouracil and cisplatin. The THUMPD1 was the target gene of miR-873-5p. Moreover, miR-873-5p could target the THUMPD1 axis so as to inhibit gastric cancer cell behavior as well as chemoresistance.

Conclusions: MiR-873-5p plays a role in regulating cell behavior as well as regulating chemoresistance in gastric cancer. In addition, THUMPD1, as a downstream molecule of miR-873-5p, plays an important role in the cell behavior and chemoresistance of gastric cancer. The research first confirmed that miR-873-5p could inhibit gastric cancer cell behavior and chemoresistance by targeting the THUMPD1.

Keywords: Gastric cancer; miR-873-5p; quantitative real-time polymerase chain reaction (qRT-PCR); Western blot

Submitted Jul 30, 2021. Accepted for publication Oct 22, 2021.

doi: 10.21037/jgo-21-641

View this article at: https://dx.doi.org/10.21037/jgo-21-641 


\section{Introduction}

Gastric cancer is one of the foremost common gastrointestinal tumors. Although the incidence rate has declined in recent years, the incidence and fatality rate still account for $8 \%$ and $10 \%$ of systemic malignancies worldwide, respectively, and most of the new cases and deaths are concentrated in East Asia and developing countries (1). In China, the incidence of gastric cancer ranks second among males and fourth among females (2). In 2017, nearly 562,000 people died of gastric cancer (3). Early diagnosis of gastric cancer is an important means to improve the treatment effect of gastric cancer and improve the prognosis of patients. Statistics show that if the disease is confined to the mucosa and submucosa in the early stage of gastric cancer treatment, the 5 -year survival rate of patients can reach more than $90 \%$ (4). However, even in developed countries, more than $50 \%$ of diagnosed patients have entered the advanced stage of the disease, and the probability that the lesion and metastasis can be completely removed by surgery is less than $50 \%$ (5). The reason is that the early symptoms of gastric cancer are hidden. As the most important diagnostic method for gastric cancer, achieving large-scale screening with gastroscopy is difficult. Moreover, gastroscopy has limitations in the diagnosis of precancerous lesions and early cancer (6).

In recent years, more studies have focused on the relationship between microRNAs (miRNAs) and gastric cancer. It is currently believed that miRNAs can participate in the occurrence, development, and metastasis of tumors by regulating cell proliferation, differentiation, apoptosis, and invasion $(7,8)$. Abnormal expression of miRNAs may lead to carcinogenic or tumor-suppressive effects in cancer, which mainly depends on the tumor type and their target genes (9). MiRNAs can also be used as oncogenes or tumor suppressor genes to promote or suppress gastric cancer development (10). For example, the expression of miR-191 in gastric cancer tissues was significantly higher than that in normal tissues, and 9 possible target genes were detected. These results suggest that miR-191 can promote the malignant transformation of gastric mucosal cells by inhibiting the expression of these genes (11). MiR$23 \mathrm{a}$ is up-regulated in gastric cancer. The protein content of IL-6R, which is the target of miR-23a, is negatively correlated with miR-23a. The above results indicate that miR-23a promotes the growth of gastric cancer cells by acting with IL-6R (12). Furthermore, previous studies confirmed that miR-206 can inhibit the expression of CyclinD2 in rhabdomyosarcoma, thereby inhibiting the occurrence of tumors (13). MiR-873-5p was first found in the hippocampus of temporal lobe epileptic rats with memory impairment (14). MiR-873-5p targets HMOX1 to regulate its expression and inhibit neuronal cell apoptosis to play a protective role (15). Meanwhile, in renal injury induced by an adhesion protein, METTL3/m6A/miRNA$873-5$ p plays a protective role by regulating the Keapl/ $\mathrm{NrG}$ pathway to reduce oxidative stress and apoptosis (16). In lung cancer, miR-873-5p is the downstream target gene of lncRNA DCGR5 to regulate the expression of TUSC3. Therefore, miR-873-5p plays an important role in tumor progression (17). However, the detailed information is still poor on the expression and biological function of miR$873-5 p$ in gastric cancer. THUMPD1 is specific adaptor protein that modulates tRNA acetylation by interacting with NAT10 which is a biomarker in some cancer types (18). Previous studies had show that THUMPD1 might interact with cancer progression and invasion, such as colon cancer, breast cancer, hepatocellular cancer, etc. (19-21).

And in our study, we also predict that the target gene of miR-873-5p is THUMPD1. So our study is the first to detect the expression of miR-873-5p in both clinical patients and cell lines of gastric cancer. Then, the functional roles of miR$873-5 p$ in the cell lines were further studied. In addition, we explored not only the detailed molecular mechanisms of miR-873-5p but also the detailed molecular mechanisms of THUMPD1. Therefore, this study may provide the basis for further relevant studies targeting gastric cancer, and provides valuable insights. We present the following article in accordance with the MDAR reporting checklist (available at https://dx.doi.org/10.21037/jgo-21-641).

\section{Methods}

\section{Tissue samples and cell lines}

From Jun. 2017 to Aug. 2019, the Department of Gastrointestinal Surgery, Zhongshan Hospital, Xiamen University collected 162 gastric cancer samples with paired normal tissues. The Zhongshan hospital ethics committee reviewed and approved the experimental protocols (Ethical Approval No. XMZSYYKY-2021-172). All processes were in accordance with the Declaration of Helsinki (as revised in 2013). Written informed consent was obtained from all participants.

Tissues were stored at $-80{ }^{\circ} \mathrm{C}$. GES, MKN28, SGC7901, AGS, MGC803, MKN7, MKN45, BGC823, and HGC27 cells were bought in advance from ATCC (Virginia). Cells 
Table 1 sequences of primers for RT-PCR

\begin{tabular}{ll}
\hline Names & Sequences (5'-3') \\
\hline miR-873-5p: Forward & GCAGGAACUUGUGAGUCUCCU \\
miR-873-5p: Reverse & AGGAGACUCACAAGUUCCUGC \\
GAPDH: Forward & GTCAGCCGCATCTTCTTT \\
GAPDH: Reverse & CGCCCAATACGACCAAAT \\
THUMPD1: forward & AAGGATCAGCAGCCCTCTGGAAGTGAG \\
THUMPD1: Reverse & CGTTGTCTTCATCAGGACACTTGGGAT \\
\hline
\end{tabular}

RT-PCR, real-time polymerase chain reaction.

were cultured in RPMI 1640 medium containing $10 \%$ Fetal Bovine Serum (FBS, Invitrogen) and incubated at 5\% $\mathrm{CO}_{2}$ and $37^{\circ} \mathrm{C}$. Six-well plates were used to seed MKN45 cells evenly. DMEM was added overnight with $10 \%$ FBS, neither with penicillin nor with streptomycin. As previously described, OPTI-MEM serum-free medium (Sigma Aldrich) as well as Lipofectamine 2000 reagent (Thermo Fisher) were adopted for transfection. In this study, siRNA with $100 \mathrm{nM}$ final concentration was used. Meanwhile, this study also employed the pEZ-Lv201 vector (Biovector) to construct the miR-873-5p overexpression system in AGS cells. The pEZLv201 vector was used as the negative control in normal AGS cells. This study also used lentiviral particles generated with standardized protocols in order to produce plasmids which were highly purified. Endo Fectin-Lenti ${ }^{\mathrm{TM}}$ and Titer Boost ${ }^{\mathrm{TM}}$ reagents (CWBio) were used to co-transfect AGS cells.

\section{qRT-PCR analysis}

Stored at $-20{ }^{\circ} \mathrm{C}$, this study extracted total RNA in each group with M5 SuperTRIgent (mei5bio). As previously described, total RNA was used to synthesize complementary DNA (cDNA) with the iScript supplementary DNA synthesis kit. The PCR reaction system and program were set up as previously described. This study adopted the $2-{ }^{\Delta \Delta \mathrm{Ct}}$ cycle method to evaluate the relative expression levels of mRNAs. The relative expression of miR-873-5p, THUMPD1 was normalized to the GAPDH value using $2^{-\Delta \Delta c t}$ values of each sample. All the Forward and Reverse primers are showed in Table 1 .

\section{Migration and invasion assay}

We used the EZCell ${ }^{\mathrm{TM}}$ cell Migration/Chemotaxis assay kit (24-well, Biovision, USA) and EZCell ${ }^{\mathrm{TM}}$ Cell Invasion
Assay (Basement Membrane) (96-well Kit) (Biovision, USA) kits to detect the migration and invasion of MKN45 cells according to the manufacturer's instructions.

\section{CCK8 assay}

Cells transfected with the overexpression system and negative control were digested, centrifuged, and resuspended. After that, cells were diluted with complete medium to 2,000 cells $/ \mathrm{mL}$. In a 96-well plate, each well was filled with $100 \mu \mathrm{L}$ of a 2,000 cells $/ \mathrm{mL}$ suspension. After sticking to the wall, the cell mediums were added Doxorubicin/Fluorouracil and cisplatin according to the gradient concentration, respectively, and then continue to culture for $48 \mathrm{~h}$. In each group, 5 replicate wells were set and were observed them in five-time points. We incubated the plate in an incubator at $37^{\circ} \mathrm{C}$ for $2 \mathrm{~h}$, and measured the absorbance at OD450. The cell proliferation was subsequently calculated.

\section{Western blot analysis}

RIPA Lysis and Extraction Buffer (Beyotime, China) was used to obtain protein from distinct cell groups. Before performing further examination with SDS-PAGE, total protein was reacted with the SDS-PAGE test buffer. Before brooding the layer with anti-Rabbit THUMPD1 (1:2,000) (Abcam, USA) and GAPDH $(1: 1,000)$ (CST, USA) for the whole night, we blocked the layer for $1 \mathrm{~h}$. After treating the proteins with the ECL chemiluminescence kit (PromoCell, Germany), and proteins were hatched at room temperature with corresponding secondary antibodies. We observed the bands.

\section{Detection of dual luciferase reporter gene}

The cells were co-transfected with THUMPD1 WT/ 
MUT plasmids or their mutant fragments and miR-873$5 \mathrm{p}$ mimic or inhibitor by using lipofectamine 3000 reagent (Invitrogen, USA) according to the protocol. The cells were lysed prior to 36-hour plasmid transfection. We also used the DLR ${ }^{\mathrm{TM}}$ Assay System kit (Promega, USA) to directly detect the expression of the luciferase reporter gene, according to the manufacturer's instructions. We used a fluorescent luminometer to measure the firefly luciferase activity. Specifically, we used $50 \mu \mathrm{L}$ of Renilla luciferase reagent to precisely detect Renilla luciferase activity. The Renilla luciferase fluorescence value and the activity ratio were considered as the internal reference and the reporter gene activity value, respectively.

\section{Statistical analysis}

SPSS16.0 statistical software was used for data analysis. The t-test was used for comparisons between the 2 groups, and one-way ANOVA was used for data comparisons between multiple groups. $\mathrm{P}$ value $<0.05$ was considered a significant difference between 2 data points.

\section{Results}

\section{MiR-873-5p expression is down-regulated in gastric cancer tissues}

We detected the expression of miR-873-5p in gastric cancer tissues and paired normal tissues. The results showed that the expression of miR-873-5p in gastric cancer tissues was significantly lower than that in normal tissues $(\mathrm{P}<0.001)$ (Figure 1A,1B). To thoroughly probe the biological function of miR-873-5p, we carefully studied miR-873-5p expression in 9 selected cell lines, including GES, MKN28, SGC7901, AGS, MGC803, MKN7, MKN45, BGC823, and HGC27. The qRT-PCR analysis showed that there were significant differences in the expression of miR-873-5p mRNA in these 9 cell lines (Figure 1C). It is noteworthy that the expression of miR-873-5p mRNA in AGS cells was significantly lower than that in other cell lines $(\mathrm{P}<0.001)$, while the expression of miR-873-5p mRNA in MKN45 cells was significantly higher than that in other cell lines $(\mathrm{P}<0.001)$. Therefore, AGS and MKN45 cells were selected for further study. In addition, we investigated the expression of miR-873-5p mRNA in gastric cancer tissues with lymph node metastasis and distant metastasis. The results suggested that miR-873$5 \mathrm{p}$ mRNA expression was significantly higher in gastric cancer tissues without distant lymph node metastasis than in gastric cancer tissues with distant lymph node metastasis $(\mathrm{P}<0.001)$ (Figure 1D,1E). We also tested the relationships between tumor volume and miR-873-5p mRNA expression. Figure $1 F$ showed that miR-873-5p mRNA expression was negatively correlated with tumor volume, suggesting that miR-873-5p may be a potential inhibitor of gastric cancer.

\section{Regulation of miR-873-5p in gastric cancer cell migration and invasion}

We performed overexpression and knockdown of miR873-5p in AGS and MKN45 cells. qRT-PCR analysis was further employed to evaluate miR-873-5p expression in AGS and MKN45 cells after different treatments. As shown in Figure 2A, miR-873-5p was successfully overexpressed and knocked down in AGS and MKN45 cells. Inhibitor \#3 and mimic \#3 were used for further study. In addition, the efficiency of miR-873-5p overexpression and knockdown in MKN45 cells and AGS cells were also detected (Figure 2B). The results showed that the adopted overexpression and knockdown systems were 2 distinct effective systems.

Moreover, we investigated the regulatory role of miR$873-5 \mathrm{p}$ in the migration and invasion of gastric cancer cells in miR-873-5p knockdown treated MKN45 cells and miR873-5p overexpression treated AGS cells. As shown in Figure 2C,2D, miR-873-5p knockdown treatment promoted cell migration and invasion compared with normal MKN45 cells $(\mathrm{P}<0.01)$. However, As shown in Figure $2 E, 2 F$, miR873-5p overexpression inhibited cell migration and invasion compared with normal AGS cells $(\mathrm{P}<0.01)$. Therefore, it is speculated that miR-873-5p mRNA expression is closely related to gastric cancer cell migration as well as cell invasion.

\section{MiR-873-5p inhibits the chemotherapy resistance of gastric cancer cells}

Three common chemotherapeutic drugs, cisplatin, doxorubicin, and fluorouracil, were used to study the effect of miR-873-5p expression changes on the chemotherapy sensitivity of gastric cancer cells. We also investigated the proliferation of miR-873-5p knockdown treated MKN45 cells and miR-873-5p overexpression treated AGS cells. As shown in Figure 3A-3C, compared with normal AGS cells, the cell survival rate of AGS cells overexpressing miR-873$5 \mathrm{p}$ was significantly reduced after treatment with the 3 different drugs $(\mathrm{P}<0.001)$. However, compared with normal MKN45 cells, miR-873-5p knockdown MKN45 cells had 
A

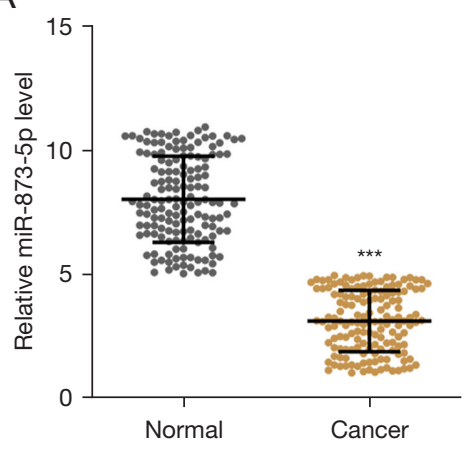

D

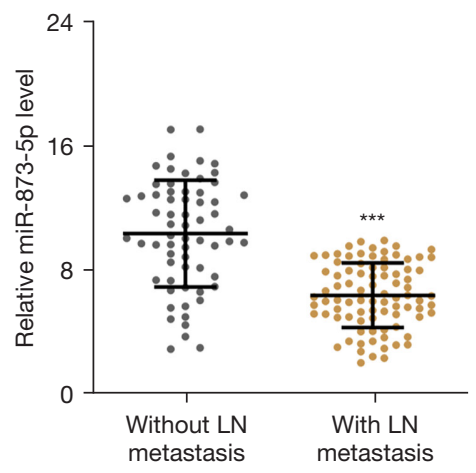

B

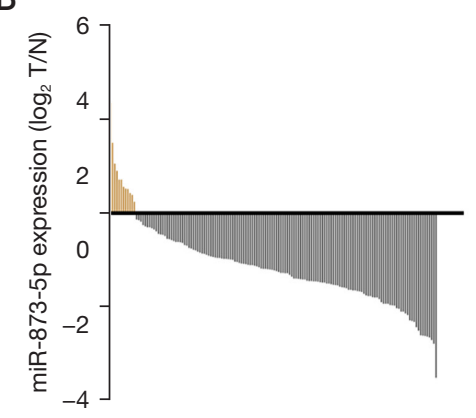

$\mathrm{E}$

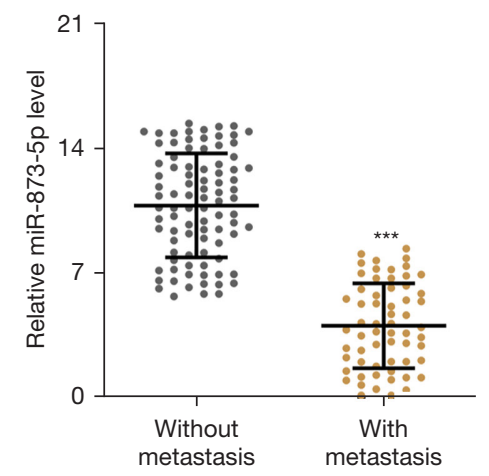

C

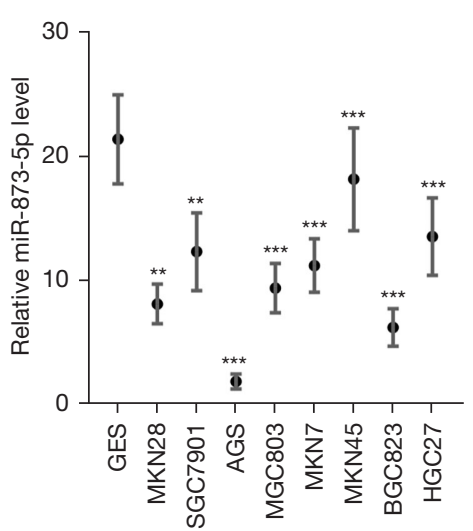

$\mathrm{F}$

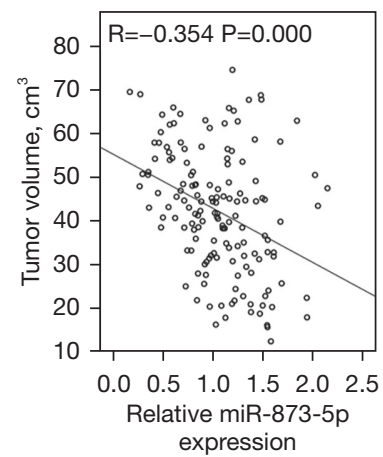

Figure $1 \mathrm{MiR}-873-5 \mathrm{p}$ expression is down-regulated in gastric cancer. (A) Analysis of the abundance of miR-873-5p in 162 tumor tissues and their paired normal tissues. (B) Analysis of the expression distribution of miR-873-5p in 162 pairs of tumor tissues and adjacent tissues. (C) Abundance analysis of miR-873-5p in 9 cell lines. (D) Abundance analysis of miR-873-5p in tumor tissues with or without lymph node metastasis. MiR-873-5p expression was up-regulated in gastric cancer tissues without lymph node metastasis. (E) Abundance analysis of miR873-5p in tumor tissues with or without metastasis. MiR-873-5p expression was up-regulated in gastric tumor tissues without metastasis. (F) Relationship analysis between miR-873-5p and tumor volume. ${ }^{* *}, \mathrm{P}<0.01 ;{ }^{* *}, \mathrm{P}<0.001$.

significantly increased viability after treatment with all 3 drugs $(\mathrm{P}<0.001)$ (Figure 3D-3F).

The above evidence shows that miR-873-5p knockdown treatment of MKN45 cells and miR-873-5p overexpression treatment of AGS cells were closely related to changes in cell viability after drug treatment.

\section{MiR-873-5p regulates THUMPD1 protein and $m R N A$ expression}

In this study, we screened 2 online databases, DIANA tools and MicroRDB, to acquire the potential targets of miR-873$5 \mathrm{p}$. The results suggested that there were 22 overlapping genes found in the 2 databases. Based on the biological functions, THUMPD1, BSCL2, PTGS1, KRT20, BPGM, TUSC3, PSG11, E2F7, PHF6, DPF2, TNRC6B, and PGM2L1 genes were selected for further analysis (Figure 4A). This study also investigated the potential relationship between the abundance of miR-873-5p and the mRNA abundance of THUMPD1, BSCL2, PTGS1, KRT20, BPGM, TUSC3, PSG11, E2F7, PHF6, DPF2, TNRC6B, and PGM2L1 genes in 162 gastric cancer samples (Figure 4B-4M).

The results showed that the expression of miR-873$5 \mathrm{p}$ was negatively correlated with the mRNA expression of THUMPD1 $(\mathrm{P}=0.006)$. Meanwhile, no significant relationships were found between miR-873-5p and BSCL2, PTGS1, KRT20, BPGM, TUSC3, PSG11, E2F7, PHF6, 
A

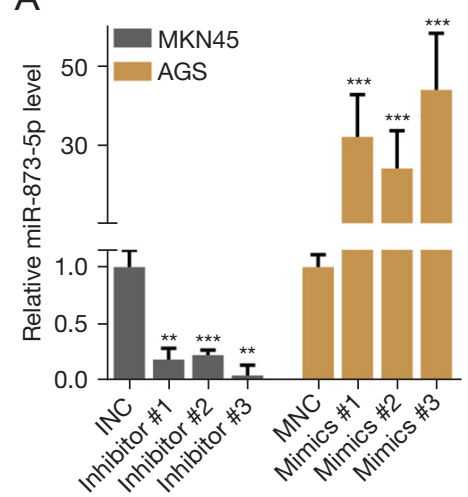

D

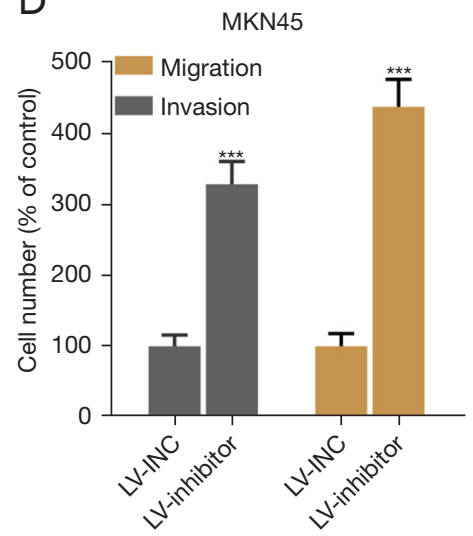

B

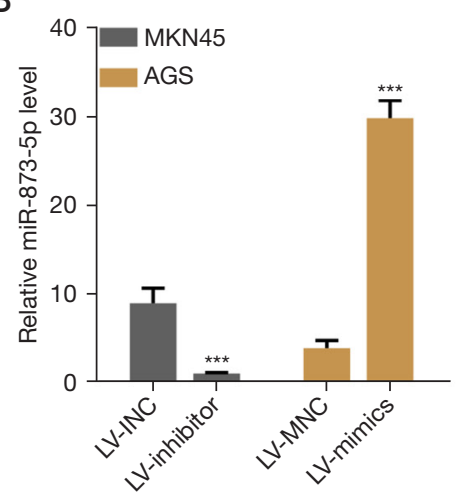

$\mathrm{E}$

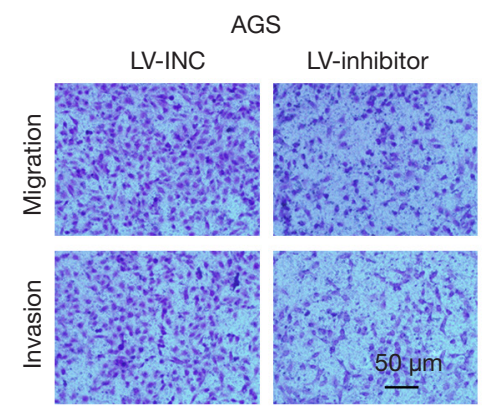

C

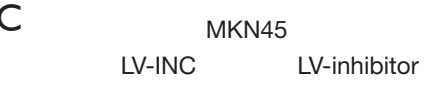

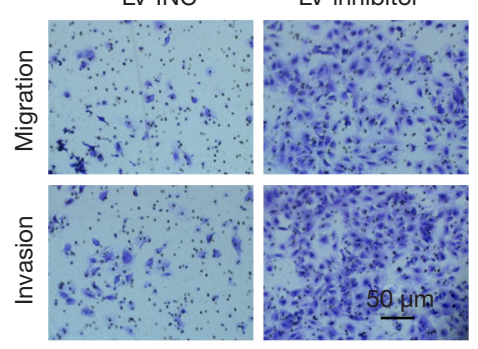

$\mathrm{F}$

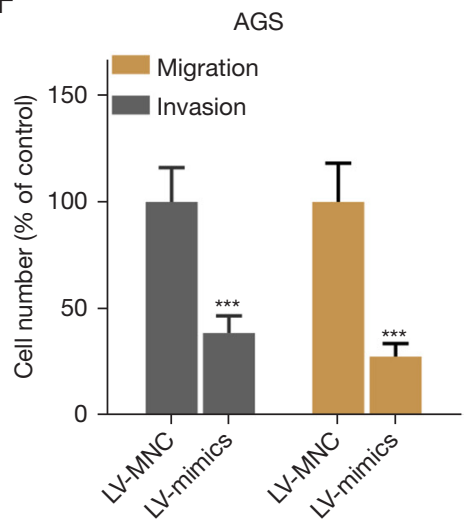

Figure 2 Regulation of miR-873-5p in gastric cancer cell migration and invasion. The invasion and migration of MKN45/AGS cells after treatment with LVs were detected based on the AKP assay. (A) qRT-PCR analysis of miR-873-5p knockdown treated MKN45 cells and miR-873-5p overexpression treated AGS cells. ${ }^{* *}, \mathrm{P}<0.01$ means inhibitor\# vs. inhibitor NC/mimics\# vs. mimicsNC, ${ }^{* * *}, \mathrm{P}<0.001$ means inhibitor\# vs. inhibitor NC/mimics\# vs. mimicsNC. (B) Inhibitor 3\# in MKN45 cells and mimics 3\# in AGS cells were further identified. ***, $\mathrm{P}<0.001$ means LV-inhibitor3 vs. LV-inhibitor NC/LV-mimics3 vs. LV-mimicsNC. (C,D) Cell migration and invasion analysis of miR873-5p knockdown treated MKN45 cells. ***, $\mathrm{P}<0.001$ means $\mathrm{LV}$-inhibitor3 vs. LV-inhibitor NC. (E,F) Cell migration and invasion analysis of miR-873-5p overexpression treated AGS cells. ${ }^{* * *}, \mathrm{P}<0.001$ means LV-mimics3 vs. LV-mimicsNC.

DPF2, TNRC6B, and PGM2L1. Therefore, THUMPD1 was solely selected to conduct further study. Moreover, as displayed in Figure $4 \mathrm{~N}$, the expression of THUMPD1 protein in AGS cells treated with miR-873-5p was significantly lower than that in normal AGS cells $(\mathrm{P}<0.001)$. THUMPD1 protein expression was significantly higher in miR-873-5p knockdown MKN45 cells than in normal MKN45 cells $(\mathrm{P}<0.001)$.

A similar phenomenon could be found in the qRTPCR analysis $(\mathrm{P}<0.001)$ (Figure $4 N)$. The above-mentioned results all suggested that miR-873-5p might affect the THUMPD1 protein as well as mRNA expression.

Furthermore, THUMPD1 mRNA 3'UTR wild type and mutant plasmids for potential binding sites of miR873-5p were constructed (Figure $5 A$ ). The DLR ${ }^{\mathrm{TM}}$ assay demonstrated that miR-873-5p could not bind to the SGPP1 mutant plasmid, but could bind to the wild type THUMPD1 mRNA 3'UTR (Figure 5B, 5C). The analysis showed that siRNA 2\# was an effective THUMPD1 gene knockdown sequence from the knockdown experiment. Therefore, AGS cells treated with siRNA 2\# were selected for further study (Figure 5D). In addition, we employed cisplatin, doxorubicin, and fluorouracil to probe the effect of THUMPD1 knockdown on the cell proliferation of miR-873-5p knockdown treated MKN45 cells. As shown in Figure 5E-5G, THUMPD1 knockdown significantly 

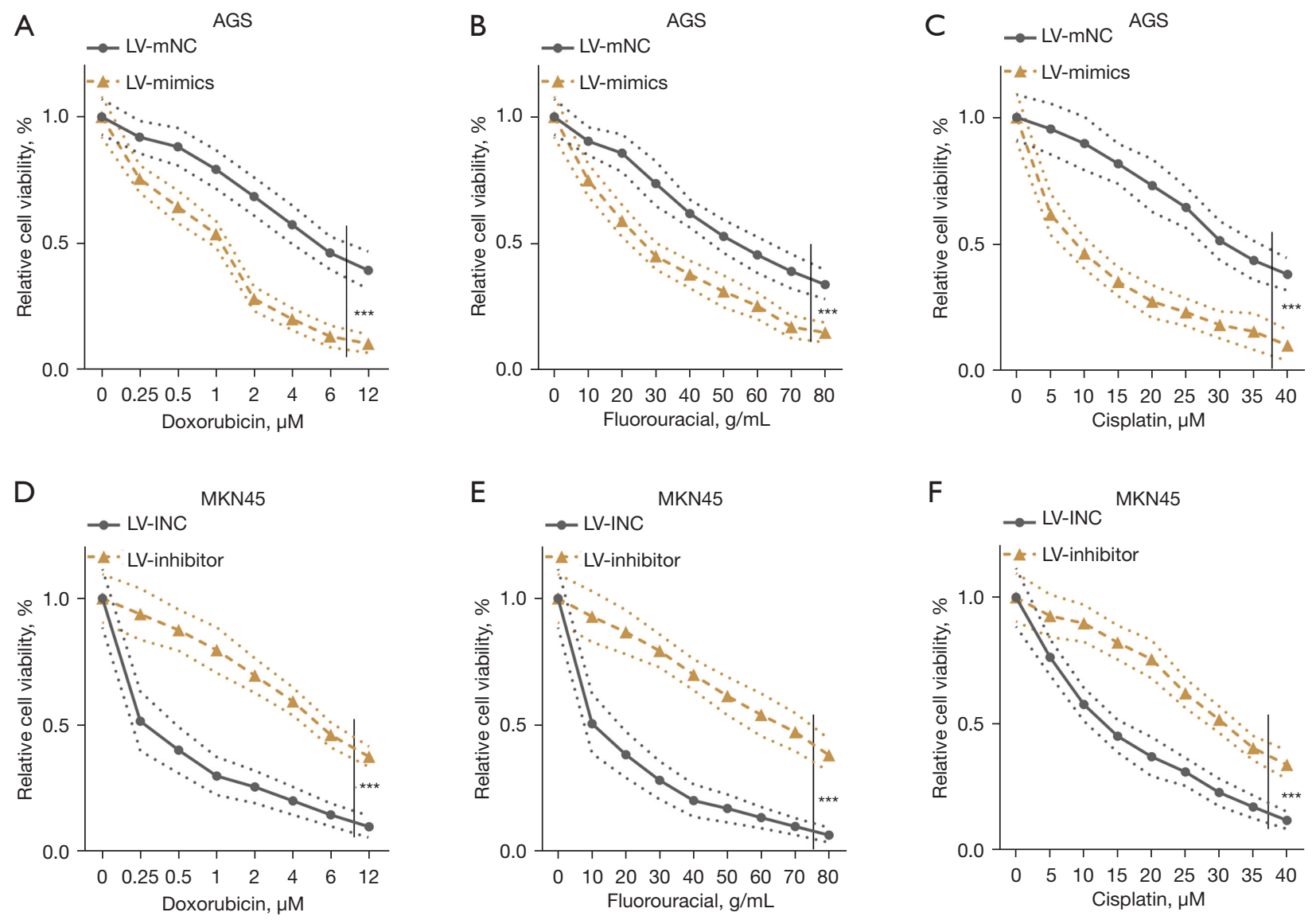

Figure 3 Effect of miR-873-5p on the chemotherapy sensitivity of gastric cancer cells. (A-C) Proliferation analysis of AGS cells overexpressing miR-873-5p after 3 chemotherapeutic drug treatments. (D-F) Proliferation analysis of MKN45 cells with miR-873-5p knockdown after 3 chemotherapy drug treatments. ***, $\mathrm{P}<0.001$.

reduced the cell survival rate in miR-873-5p knockdown MKN45 cells compared with miR-873-5p knockdown MKN45 cells $(\mathrm{P}<0.001)$.

Altogether, these results revealed that THUMPD1 knockdown in miR-873-5p knockdown MKN45 cells was closely related to changes in cell viability induced by drug therapy.

\section{Discussion}

Exploring and identifying targets for malignant tumor treatment is a crucial topic for cancer research. Gastric cancer, a highly malignant disease with high mortality, has a poor prognosis (22). Currently, although the strategies to prevent gastric cancer are gradually becoming more mature, obstacles and difficulties still exist in the early diagnosis of gastric cancer (23). With the in-depth study of the epidemiology of gastric cancer, it is now widely accepted that the occurrence of gastric cancer appears to be a complex process including multiple factors, multiple genes, and multiple steps (24). Notably, the relationship between miRNAs and gastric cancer has attracted growing attention from researchers in recent years (25). MiRNAs are highly conserved small molecule single-stranded RNAs, which regulate the expression of the target gene by base pairing with the target gene mRNA to guide its degradation or hinder its translation (26). Previous studies have shown that multiple miRNAs are related to gastric cancer, including miR-21, miR-21, miR-29, miR-106, miR-let7a, miR-148, and miR-622, among others. MiR-21, miR-10b, miR-2223, miR-338, miR-let7a, have been confirmed to be tumor markers for evaluating the prognosis of gastric cancer (27). Meanwhile, miR-212 and miR-195 have been proven to be closely related to lymph node metastasis (28). Therefore, 
A

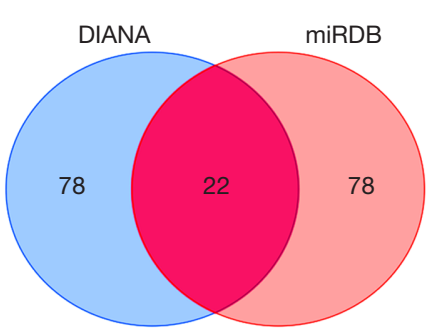

D

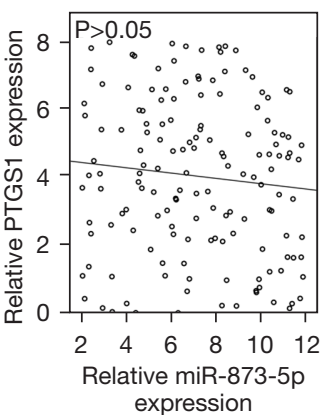

$\mathrm{H}$

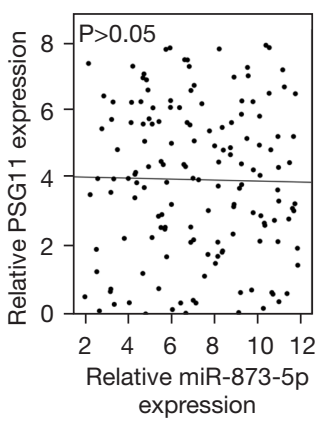

$\mathrm{L}$

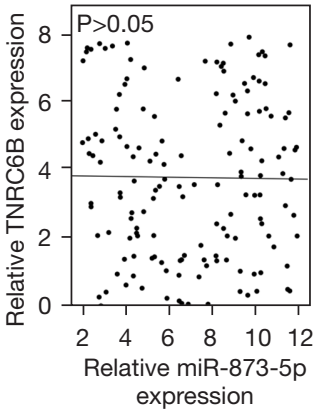

E

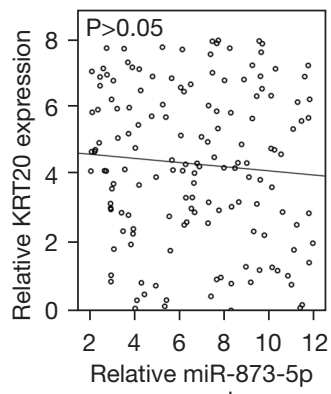

expression

I

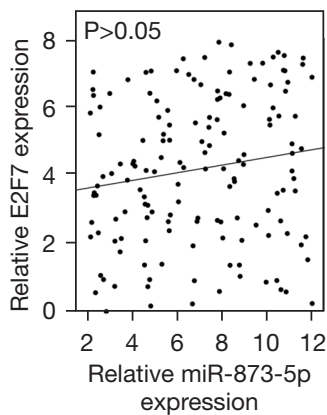

M

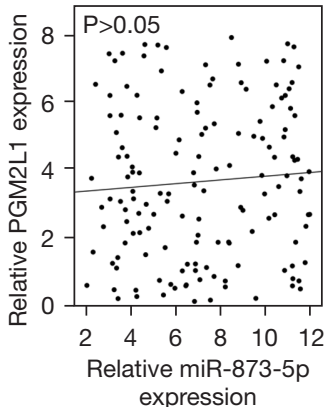

B

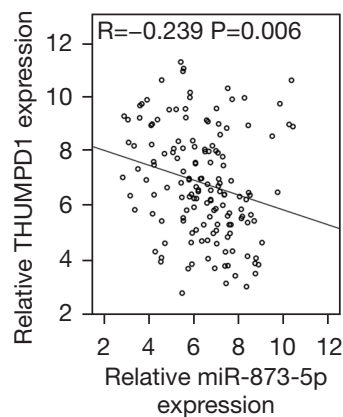

F

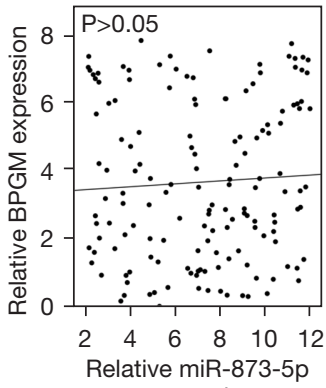

expression

J

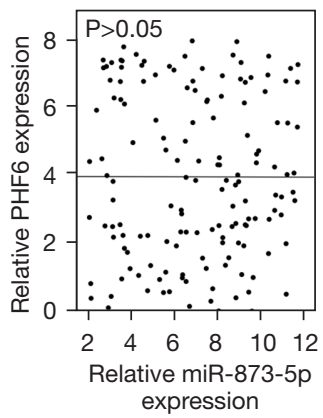

$\mathrm{N}$

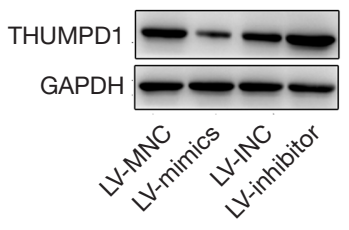

C

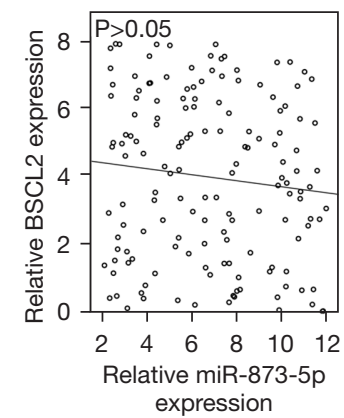

G

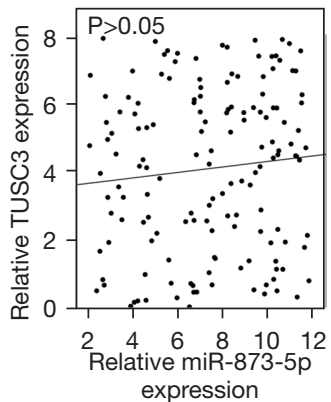

K
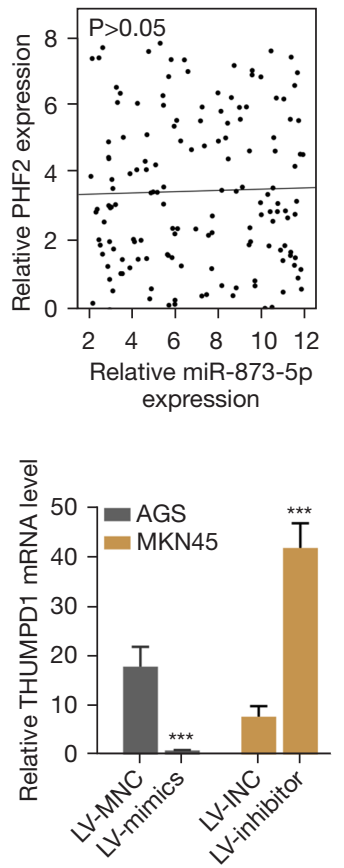

Figure 4 MiR-873-5p plays an important role in gastric cancer through THUMPD1. (A) Venn diagram analysis of potential target genes of miR-873-5p in 2 databases. (B-M) Correlation analysis of miR-873-5p abundance and the mRNA expression of THUMPD1, BSCL2, PTGS1, KRT20, BPGM, TUSC3, PSG11, E2F7, PHF6, DPF2, TNRC6B, and PGM2L1. (N) The qRT-PCR and western blot analysis of THUMPD1 expression in miR-873-5p overexpression treated AGS cells and miR-873-5p knockdown treated MKN45 cells. ***, $\mathrm{P}<0.001$. 
A

THUMPD1 3'UTR WT1 ...TTAGGGGTTTCCTGAATTTGAAA...

THUMPD1 3'UTR WT2 ...TTTCATAGTTCCTGATATTTTCA...

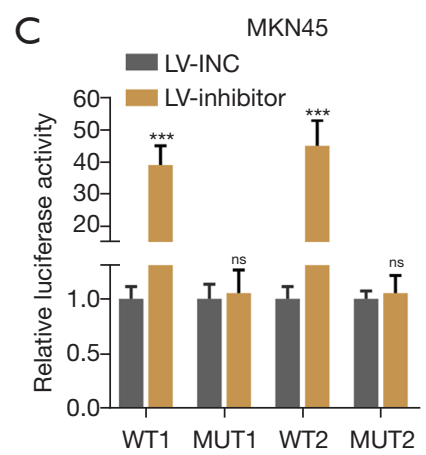

$E$

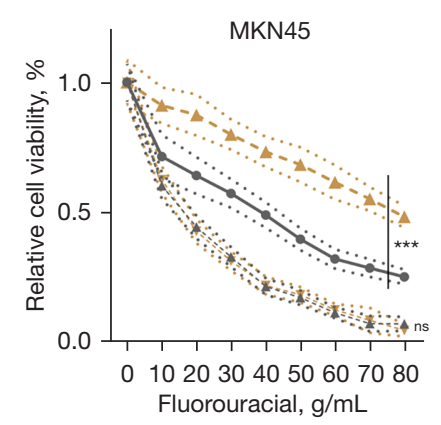

B
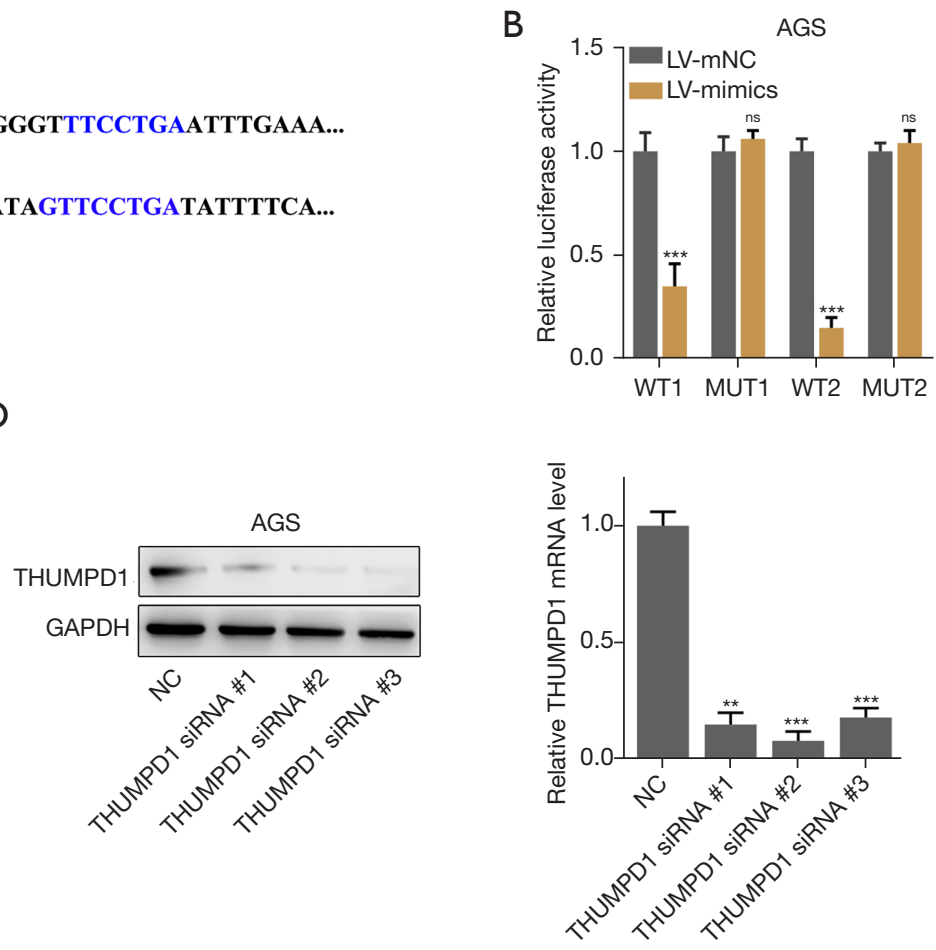

G

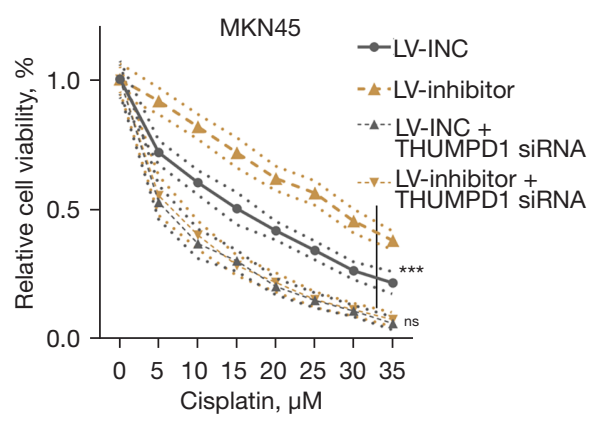

Figure 5 MiR-873-5p directly binds to the 3'UTR of THUMPD1 mRNA. (A) Potential binding sites between miR-873-5p and the 3'UTR of THUMPD1 mRNA. (B,C) Dual luciferase reporter gene analysis of the binding sites between miR-873-5p, wild type, and mutation of the 3'UTR in THUMPD1 mRNA. (D) Protein and mRNA analysis of the THUMPD1 knockdown effect in AGS cells. (E-G) Cell viability analysis after treatment with 3 chemotherapy drugs in miR-873-5p and THUMPD1 knockdown treated MKN45 cells. ***, $\mathrm{P}<0.001$.

novel tumor markers are particularly important for the early diagnosis and individualized treatment of patients.

Previous studies have shown that miR-873-5p is involved in the pathogenesis of a variety of tumors, and is downregulated in glioblastoma, lung adenocarcinoma, and colorectal cancer (29-31). However, the role of miR-873$5 \mathrm{p}$ in gastric cancer remains unknown. The findings of this study suggested that the alteration of miR-873-5p expression might be closely related to the cell proliferation as well as the chemoresistance of gastric cancer. Therefore, miR-873-5p may appear to be a potential target for gastric cancer treatment. Although the hidden mechanism of miR$873-5 \mathrm{p}$ in patients with gastric cancer may be complicated and diversified, it still appears to be a molecule that should not be ignored in tumorigenesis and development. This study demonstrated that the expression of miR-873-5p in gastric cancer was lower than that in adjacent tissues. Either the overexpression or knockout of miR-873-5p 
could affect the migration, invasion, and chemoresistance of gastric cancer. It is therefore suggested that miR-873$5 p$ dysfunction appears to be an influencing factor for the progression of gastric cancer.

Human THUMPD1 is a specific adaptor protein, which is able to interact with NAT10 for the regulation of tRNA acetylation (18). The study of Havugimana et al. demonstrated that THUMPD1 is capable of interacting with Yes-related protein (YAP) (32). YAP plays a role as the main transcriptional co-activator of the Hippo signaling pathway, which is vital in tumor proliferation and tumor invasion (33-34). In addition, a previous study indicated that the invasion and migration of breast cancer cells are promoted by THUMPD1 through the AKT-GSK3 $\beta$-snail pathway (35). The PI3K/Akt signaling pathway counts as one of the most important signal transduction pathways in cells, which plays a role in the regulation of multiple important biological processes. This signaling pathway has comparatively high status in inhibiting apoptosis as well as promoting proliferation by influencing the activation of downstream effector molecules (36). Studies have also illustrated that the PI3K/Akt signaling pathway could help with tumor invasion as well as metastasis by regulating EMT. For example, Grille et al. discovered that the PI3K/ Akt signaling pathway participated in the process of induction of EMT in squamous cell carcinoma cells, and could accelerate the invasion and metastasis of tumor cells (37). GSK3 $\beta$ is a multifunctional kinase composed of serine and threonine, which help with regulating glycogen metabolism. It is a downstream gene of the PI3K/Akt signaling pathway and can phosphorylate snail transcription factor to regulate EMT (38). Bai et al. suggested that OLA1 regulates EMT through GSK3 $3 /$ snail/E-cadherin in lung cancer, thus regulating the invasion and metastasis of lung cancer (39). Furthermore, the PI3K/Akt/GSK3 $\beta$ signaling pathway has also been shown to regulate EMT in breast cancer and gastric cancer $(35,40)$. Therefore, as a downstream gene of the PI3K/Akt signaling pathway, GSK3 $\beta$ can phosphorylate snail to regulate EMT and participate in tumor invasion and metastasis. In this study, our results showed that the expression level of THUMPD1 in gastric cancer is closely related to miR-873-5p. Meanwhile, THUMPD1 and miR-873-5p are important in chemotherapy resistance, migration, and invasion. Based on the evidence acquired in this study, we can reasonably speculate that miR-873-5p plays an important role in THUMPD1 regulation, and notably, THUMPD1 seems to be vital in the chemotherapy resistance and functions of gastric cancer cells.

\section{Conclusions}

In summary, we demonstrated the expression of miR-873$5 \mathrm{p}$ in gastric cancer tissues and cells was downregulated. MiR-873-5p could regulate gastric cancer cell migration, invasion, and chemotherapy resistance through the THUMPD1 axis. Our research first confirmed that miR873-5p could inhibit gastric cancer cell behavior and chemoresistance by targeting the THUMPD1. The results might help with future studies relevant to gastric cancer by providing detailed information.

\section{Acknowledgments}

We thank Shanghai Tongshu Biotechnology Co., Ltd for technical support.

Funding: None.

\section{Footnote}

Reporting Checklist: The authors have completed the MDAR reporting checklist. Available at https://dx.doi. org/10.21037/jgo-21-641

Data Sharing Statement: Available at https://dx.doi. org/10.21037/jgo-21-641

Conflicts of Interest: All authors have completed the ICMJE uniform disclosure form (available at https://dx.doi. org/10.21037/jgo-21-641). All authors reported that they received technical support from Shanghai Tongshu Biotechnology Co., Ltd. The authors have no other conflicts of interest to declare.

Ethical Statement: The authors are accountable for all aspects of the work in ensuring that questions related to the accuracy or integrity of any part of the work are appropriately investigated and resolved. The Zhongshan hospital ethics committee reviewed and approved the experimental protocols (Ethical Approval No. XMZSYYKY-2021-172). All processes were in accordance with the Declaration of Helsinki (as revised in 2013). Written informed consent was obtained from all participants.

Open Access Statement: This is an Open Access article 
distributed in accordance with the Creative Commons Attribution-NonCommercial-NoDerivs 4.0 International License (CC BY-NC-ND 4.0), which permits the noncommercial replication and distribution of the article with the strict proviso that no changes or edits are made and the original work is properly cited (including links to both the formal publication through the relevant DOI and the license). See: https://creativecommons.org/licenses/by-nc-nd/4.0/.

\section{References}

1. Siegel RL, Miller KD, Jemal A. Cancer statistics, 2019. CA Cancer J Clin 2019;69:7-34.

2. Rawla P, Barsouk A. Epidemiology of gastric cancer: global trends, risk factors and prevention. Prz Gastroenterol 2019;14:26-38.

3. GBD 2017 Stomach Cancer Collaborators. The global, regional, and national burden of stomach cancer in 195 countries, 1990-2017: a systematic analysis for the Global Burden of Disease study 2017. Lancet Gastroenterol Hepatol 2020;5:42-54.

4. Ikeda Y, Saku M, Kishihara F, et al. Effective follow-up for recurrence or a second primary cancer in patients with early gastric cancer. Br J Surg 2005;92:235-9.

5. Hohenberger P, Gretschel S. Gastric cancer. Lancet 2003;362:305-15.

6. Zhu L, Qin J, Wang J, et al. Early Gastric Cancer: Current Advances of Endoscopic Diagnosis and Treatment. Gastroenterol Res Pract 2016;2016:9638041.

7. Wu SR, Wu Q, Shi YQ. Recent advances of miRNAs in the development and clinical application of gastric cancer. Chin Med J (Engl) 2020;133:1856-67.

8. Gao M, Yin H, Fei ZW. Clinical application of microRNA in gastric cancer in Eastern Asian area. World J Gastroenterol 2013;19:2019-27.

9. Peng Y, Croce CM. The role of MicroRNAs in human cancer. Signal Transduct Target Ther 2016;1:15004.

10. Wu WK, Lee CW, Cho CH, et al. MicroRNA dysregulation in gastric cancer: a new player enters the game. Oncogene 2010;29:5761-71.

11. Peng WZ, Ma R, Wang F, et al. Role of miR-191/425 cluster in tumorigenesis and diagnosis of gastric cancer. Int J Mol Sci 2014;15:4031-48.

12. Zhu LH, Liu T, Tang H, et al. MicroRNA-23a promotes the growth of gastric adenocarcinoma cell line MGC803 and downregulates interleukin-6 receptor. FEBS J 2010;277:3726-34.

13. Novák J, Kružliak P, Bienertová-Vašků J, et al.
MicroRNA-206: a promising theranostic marker. Theranostics 2014;4:119-33.

14. Liu X, Wu Y, Huang Q, et al. Grouping PentylenetetrazolInduced Epileptic Rats According to Memory Impairment and MicroRNA Expression Profiles in the Hippocampus. PLoS One 2015;10:e0126123.

15. Shi R, Zhang S, Cheng G, et al. Ginsenoside Rg1 and Acori Graminei Rhizoma Attenuates Neuron Cell Apoptosis by Promoting the Expression of miR-873-5p in Alzheimer's Disease. Neurochem Res 2018;43:1529-38.

16. Wang J, Ishfaq $M, X u L$, et al. METTL3/m6A/miRNA873-5p Attenuated Oxidative Stress and Apoptosis in Colistin-Induced Kidney Injury by Modulating Keap1/ Nrf2 Pathway. Front Pharmacol 2019;10:517.

17. Luo J, Zhu H, Jiang H, et al. The effects of aberrant expression of LncRNA DGCR5/miR-873-5p/TUSC3 in lung cancer cell progression. Cancer Med 2018;7:3331-41.

18. Sharma S, Langhendries JL, Watzinger P, et al. Yeast Kre33 and human NAT10 are conserved 18S rRNA cytosine acetyltransferases that modify tRNAs assisted by Tan1/THUMPD1. Nucleic Acids Res 2015;43:2242-58.

19. Ma R, Chen J, Jiang S, et al. Up regulation of NAT10 promotes metastasis of hepatocellular carcinoma cells through epithelial-to-mesenchymal transition. Am J Transl Res 2016;8:4215-23.

20. Zhang H, Hou W, Wang HL, et al. GSK-3ß-regulated $\mathrm{N}$-acetyltransferase 10 is involved in colorectal cancer invasion. Clin Cancer Res 2014;20:4717-29.

21. Zhang X, Jiang G, Sun M, et al. Cytosolic THUMPD1 promotes breast cancer cells invasion and metastasis via the AKT-GSK3-Snail pathway. Oncotarget 2017;8:13357-66.

22. Fontana E, Smyth EC. Novel targets in the treatment of advanced gastric cancer: a perspective review. Ther Adv Med Oncol 2016;8:113-25.

23. Lee JH, Kim KM, Cheong JH, et al. Current management and future strategies of gastric cancer. Yonsei Med J 2012;53:248-57.

24. Shi J, Qu YP, Hou P. Pathogenetic mechanisms in gastric cancer. World J Gastroenterol 2014;20:13804-19.

25. Hao NB, He YF, Li XQ, et al. The role of miRNA and lncRNA in gastric cancer. Oncotarget 2017;8:81572-82.

26. Macfarlane LA, Murphy PR. MicroRNA: Biogenesis, Function and Role in Cancer. Curr Genomics 2010;11:537-61.

27. Li X, Zhang Y, Zhang Y, et al. Survival prediction of gastric cancer by a seven-microRNA signature. Gut 2010;59:579-85.

28. Wu WY, Xue XY, Chen ZJ, et al. Potentially predictive 
microRNAs of gastric cancer with metastasis to lymph node. World J Gastroenterol 2011;17:3645-51.

29. Cui J, Yang Y, Li H, et al. MiR-873 regulates ER $\alpha$ transcriptional activity and tamoxifen resistance via targeting CDK3 in breast cancer cells. Oncogene 2015;34:3895-907.

30. Wang RJ, Li JW, Bao BH, et al. MicroRNA-873 (miRNA-873) inhibits glioblastoma tumorigenesis and metastasis by suppressing the expression of IGF2BP1. J Biol Chem 2015;290:8938-48.

31. Gao Y, Xue Q, Wang D, et al. miR-873 induces lung adenocarcinoma cell proliferation and migration by targeting SRCIN1. Am J Transl Res 2015;7:2519-26.

32. Havugimana PC, Hart GT, Nepusz T, et al. A census of human soluble protein complexes. Cell 2012;150:1068-81.

33. Kim HM, Jung WH, Koo JS. Expression of Yes-associated protein (YAP) in metastatic breast cancer. Int J Clin Exp Pathol 2015;8:11248-57.

34. Wang Y, Xie C, Li Q, et al. Clinical and prognostic significance of Yes-associated protein in colorectal cancer. Tumour Biol 2013;34:2169-74.

35. Zhang X, Jiang G, Sun M, et al. Cytosolic THUMPD1

Cite this article as: Chen Q, Lin L, Xiong B, Yang W, Huang J, Shi H, Wang Z. MiR-873-5p targets THUMPD1 to inhibit gastric cancer cell behavior and chemoresistance. J Gastrointest Oncol 2021;12(5):2061-2072. doi: 10.21037/jgo-21-641 promotes breast cancer cells invasion and metastasis via the AKT-GSK3-Snail pathway. Oncotarget 2017;8:13357-66.

36. Jiang N, Dai Q, Su X, et al. Role of PI3K/AKT pathway in cancer: the framework of malignant behavior. Mol Biol Rep 2020;47:4587-629.

37. Grille SJ, Bellacosa A, Upson J, et al. The protein kinase Akt induces epithelial mesenchymal transition and promotes enhanced motility and invasiveness of squamous cell carcinoma lines. Cancer Res 2003;63:2172-8.

38. Hermida MA, Dinesh Kumar J, Leslie NR. GSK3 and its interactions with the PI3K/AKT/mTOR signalling network. Adv Biol Regul 2017;65:5-15.

39. Bai L, Yu Z, Zhang J, et al. OLA1 contributes to epithelialmesenchymal transition in lung cancer by modulating the GSK3 $\beta /$ snail/E-cadherin signaling. Oncotarget 2016;7:10402-13.

40. Dai J, Qian C, Su M, et al. Gastrokine-2 suppresses epithelial mesenchymal transition through PI3K/ AKT/GSK3 $\beta$ signaling in gastric cancer. Tumour Biol 2016;37:12403-10. 